\title{
Enhancement of Efficiency and Lifetime of Blue Organic Light-Emitting Diodes Using Two Dopants in Single Emitting Layer
}

\author{
Jianning Yu, ${ }^{1,2}$ Na Wei, ${ }^{1}$ Chong Li, ${ }^{3}$ Bin Wei, ${ }^{1}$ Wei Huang, ${ }^{3}$ Taiju Tsuboi, ${ }^{4}$ \\ Jianhua Zhang, ${ }^{2}$ and Zhilin Zhang ${ }^{1}$ \\ ${ }^{1}$ Key Laboratory of Advanced Display and System Applications, Shanghai University, Ministry of Education, \\ Yanchang Road 149, Shanghai 200072, China \\ ${ }^{2}$ School of Mechanical and Electronic Engineering and Automation, Shanghai University, Shanghai 200072, China \\ ${ }^{3}$ Key Laboratory for Organic Electronics and Information Displays and Institute of Advanced Materials, \\ Nanjing University of Posts and Telecommunications, Wenyuan Road 9, Nanjing 210046, China \\ ${ }^{4}$ Faculty of Engineering, Kyoto Sangyo University, Kamigamo, Kita-ku, Kyoto 603-8555, Japan
}

Correspondence should be addressed to Chong Li, li.chong@wxrtc.com and Bin Wei, bwei@shu.edu.cn

Received 9 February 2012; Revised 8 April 2012; Accepted 24 April 2012

Academic Editor: Saulius Grigalevicius

Copyright (C) 2012 Jianning Yu et al. This is an open access article distributed under the Creative Commons Attribution License, which permits unrestricted use, distribution, and reproduction in any medium, provided the original work is properly cited.

We have demonstrated efficient blue organic light-emitting diode with the structure of indium tin oxide/4,4', $4^{\prime \prime}-$ tris $(N-(2-$ naphthyl)- $N$-phenyl-amino)triphenylamine/1,4-bis[ $N$-(1-naphthyl)- $N^{\prime}$-phenylamino]-4,4'-diamine/9,10-di(2-naphthyl)anthracene (ADN): 1-4-di-[4-(N,N-di-phenyl)amino]styryl-benzene (DSA-ph) $3 \mathrm{wt} \% /$ tris-(8-hydroxyquinoline)aluminum/LiF/Al. Improved efficiencies and longer operational lifetime were obtained by codoping a styrylamine-based dopant BD-3 (0.1 wt $\%)$ into the emitting layer of ADN doped with DSA-ph compared to the case of non-codoping. This was due to the improved charge balance and expansion of exciton recombination zone. The better charge balance was obtained by reducing the electron mobility of ADN which was higher than the hole mobility in the case of non-codoping.

\section{Introduction}

High performance and high efficiency in organic lightemitting diodes (OLEDs) depend on charge carrier distribution and charge balance. Especially the charge balance is very important to achieve a long lifetime of OLED, because the balance avoids charge leakage from the emitting layer to the electrodes. A number of methods have been undertaken to achieve high carrier density and charge balance. For example, one method is to carry out a good alignment between the Fermi level of the electrode and the corresponding transport band of the adjacent organic layer. Other methods are that indium tin oxide (ITO) has been physically (e.g., UVozone), chemically, and/or electrochemically treated, and hole injection layer (HIL) has been inserted between anode and organic layer, to reduce the carrier injection barrier height and to obtain high-density hole injection [1].
The electron mobility is largely different from the hole mobility for most organic materials, giving rise to charge imbalance. It is necessary to make the electron and hole mobilities in emitting layer (EML) comparable to each other [2]. In the present paper we propose a codoping method to obtain the comparable mobilities using a blue OLED.

Blue OLEDs are important for various applications, for example, traffic signals, full color scanners, displays, and lighting including white emission generated by color conversion method. Therefore we fabricate a blue OLED to find a method to obtain the charge-balanced OLEDs. Of various blue emitters, 1-4-di-[4-(N,N-di-phenyl)amino]styrylbenzene (DSA-ph) has been paid much attention because of its high efficiency and stability [3]. Diphenylanthracene derivative, 9,10-di(2-naphthyl)anthracene (ADN) has been used as highly efficient host material for not only green 


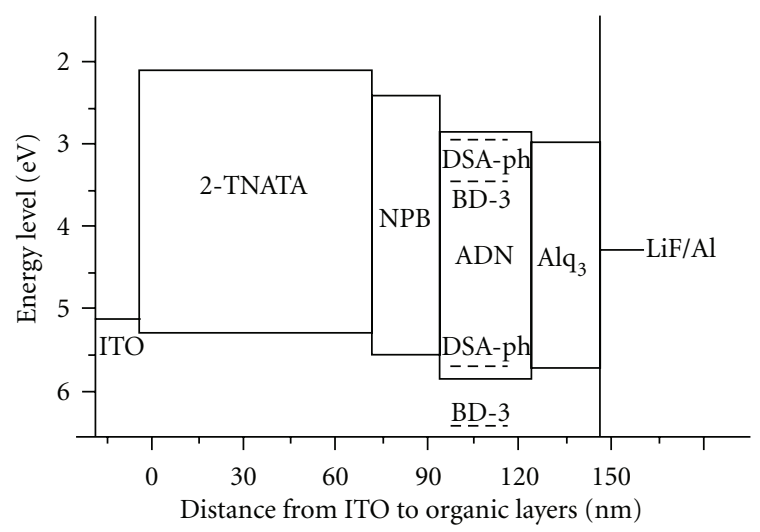

Figure 1: Device structure and energy levels of Device B.

and red emitters but also blue emitters such as 2,5,8,11tetra-t-butylperylene (TBP) [4] and 4,4'-bis[2-(4-(N,Ndiphenylamino)phenyl)vinyl]biphenyl (DPAVBi) [5]. Therefore we are concerned with blue OLED with emitting layer (EML) of DSA-ph doped ADN in this paper.

\section{Experimental}

The OLEDs were fabricated by vacuum thermal evaporation method. The base pressure was better than $7 \times 10^{-4} \mathrm{~Pa}$ during the evaporation of organic materials and $\mathrm{Al}$ cathode. The deposition rate was lower than $0.3 \mathrm{~nm} / \mathrm{s}$ for $4,4^{\prime}$, $4^{\prime \prime}$-tris $(N$-(2-naphthyl)- $N$-phenyl-amino) triphenylamine (2-TNATA), 1,4-bis[N-(1-naphthyl)- $N^{\prime}$-phenylamino]-4,4' diamine (NPB), ADN, DSA-ph, and tris-(8-hydroxyquinoline)aluminum $\left(\mathrm{Alq}_{3}\right)$. All these materials were purchased from e-Ray Optoelectronics Technology Co., Ltd. All the device fabrication processes were performed without breaking the vacuum. The layer thickness was controlled in situ using a quartz crystal monitor.

The following two devices were fabricated:

Device A: ITO/2-TNATA $(75 \mathrm{~nm}) / \mathrm{NPB}(20 \mathrm{~nm}) /$ ADN: DSA-ph $3 \mathrm{wt} \% \quad(30 \mathrm{~nm}) / \mathrm{Alq}_{3} \quad(20 \mathrm{~nm}) / \mathrm{LiF}$ $(0.5 \mathrm{~nm}) / \mathrm{Al}(100 \mathrm{~nm})$;

Device B: ITO/2-TNATA $(75 \mathrm{~nm}) / \mathrm{NPB} \quad(20 \mathrm{~nm}) /$ ADN: DSA-ph $3 \mathrm{wt} \%$ : BD-3 $0.1 \mathrm{wt} \%(30 \mathrm{~nm}) / \mathrm{Alq}_{3}$ $(20 \mathrm{~nm}) / \mathrm{LiF}(0.5 \mathrm{~nm}) / \mathrm{Al}(100 \mathrm{~nm})$,

where the thickness of each layer and the doping concentration of emitting layer (EML) are indicated. The ITO had a thickness of $220 \mathrm{~nm}$. The layer structure of Device B is shown in Figure 1, together with the highest occupied molecular orbital (HOMO) and lowest unoccupied molecular orbital (LUMO) energy levels for each layer.

The difference of Device B from Device A is that a styrylamine-based blue-emitting dye BD-3 is codoped in EML in Device B. The BD-3 was purchased from Kodak Co., Ltd. It was selected because it exhibited a very high electroluminescence (EL) efficiency of $7.2 \mathrm{~cd} / \mathrm{A}$ and emitted a $448 \mathrm{~nm}$ deep blue light which can be distinguished from DSA-ph emission $[6]$.

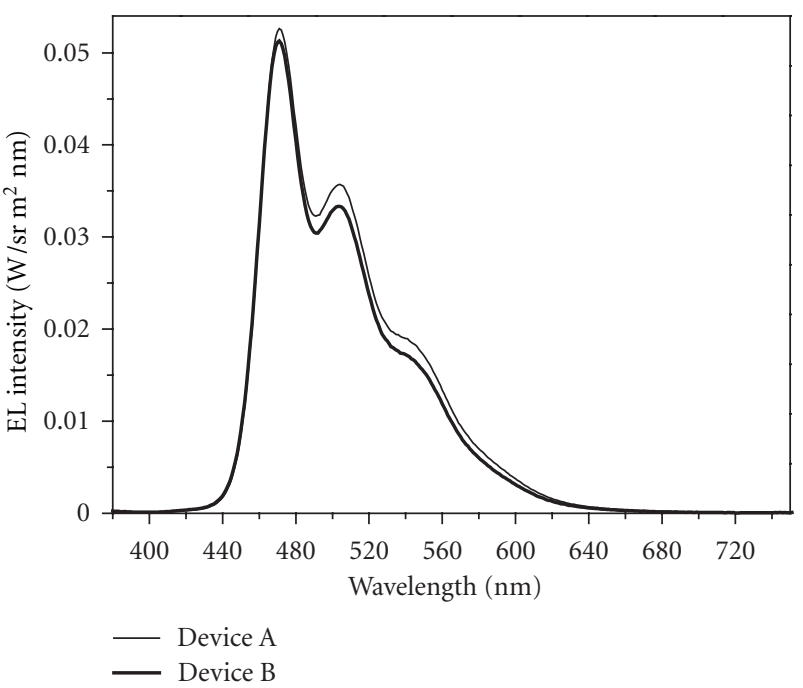

FIGURE 2: Electroluminescence spectra of Devices A and B measured at $10 \mathrm{~V}$.

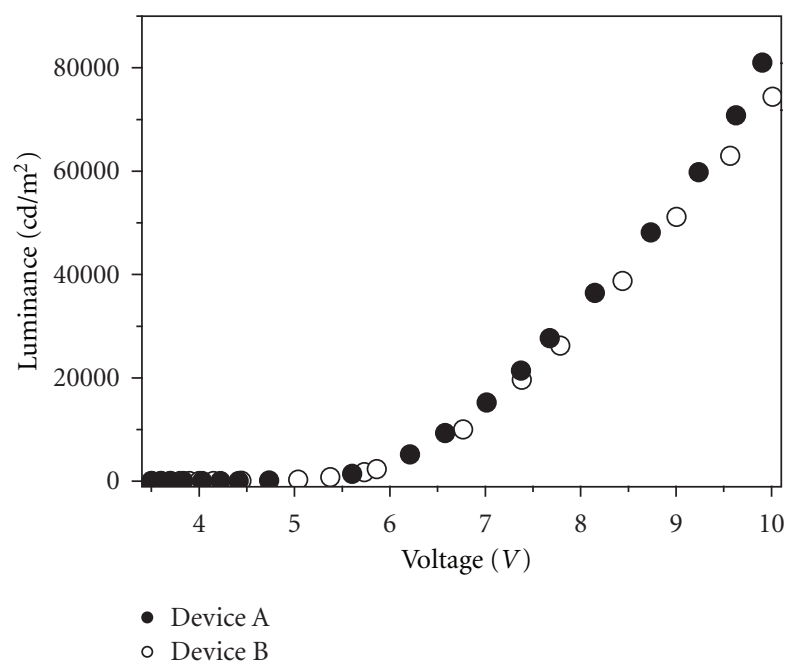

Figure 3: The luminance-voltage characteristics of Devices A and B.

\section{Results and Discussion}

Figure 2 shows the EL spectra of Devices A and B measured at $10 \mathrm{~V}$. The nearly identical EL spectra were obtained for the two devices, that is, intense peak at $470 \mathrm{~nm}$ accompanied by vibronic sidebands at 504 and about $540 \mathrm{~nm}$, but a higher intensity for Device A than for Device B. The $470 \mathrm{~nm}$ emission band with two vibronic sidebands is ascribed to DSAph. Emission of ADN at 452-455 nm [7-9] was not observed, indicating efficient energy transfer from ADN to the dopant. Emission of BD-3 at $448 \mathrm{~nm}$ [10] was also not observed because of much smaller concentration than DSA-ph.

The luminance-voltage $(L-V)$ and current densityvoltage $(J-V)$ characteristics are shown in Figures 3 and 4, respectively. It is found that the luminance is higher for Device A than for Device B at high voltages above about $6.5 \mathrm{~V}$ (Figure 3). Different OLED performance is observed between 


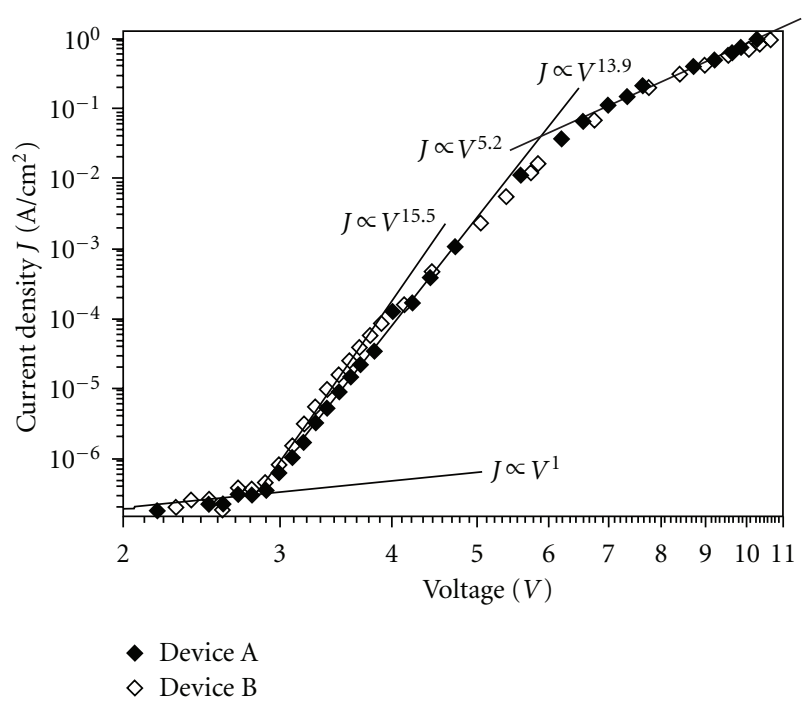

Figure 4: The current density-voltage characteristics of Devices A and $\mathrm{B}$.

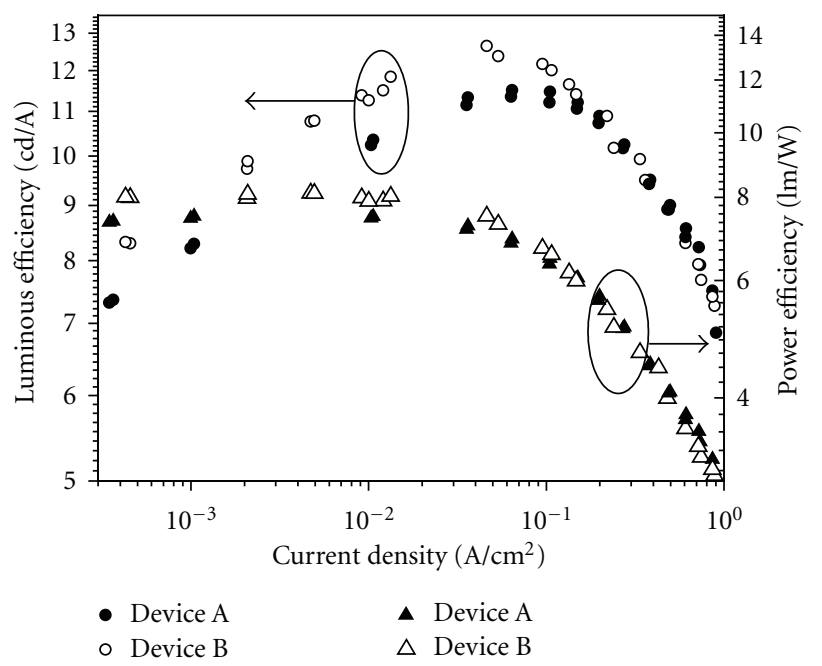

Figure 5: The luminous power efficiencies of Devices A and B, which are plotted against current density.

the two devices although the two devices follow the same Ohmic law of $J \propto V$ below $3 \mathrm{~V}$ and $J \propto V^{5.2}$ above $7 \mathrm{~V}$, while Devices $\mathrm{A}$ and $\mathrm{B}$ follow $J \propto V^{13.9}$ and $J \propto V^{15.5}$ at 3-4 V, respectively (Figure 4). An increase of current density in Device B relative to Device A is observed at $3-4 \mathrm{~V}$. This indicates increase of electron mobility due to decrease of electron traps in Device B.

Figure 5 shows the luminous efficiency-current density $\left(\eta_{\text {lumi }}-J\right)$ and the power efficiency-current density $\left(\eta_{\text {power }}-\right.$ $J)$ characteristics for the two devices. The efficiencies $\eta_{\text {lumi }}$ and $\eta_{\text {power }}$ of Device $\mathrm{B}$ are higher than those of Device $\mathrm{A}$ at current densities below $0.1 \mathrm{~A} / \mathrm{cm}^{2}$, while these efficiencies are almost the same at current densities above $0.2 \mathrm{~A} / \mathrm{cm}^{2}$ for the two devices. The external quantum efficiency (EQE) of fluorescent OLEDs is considered to be the product of the

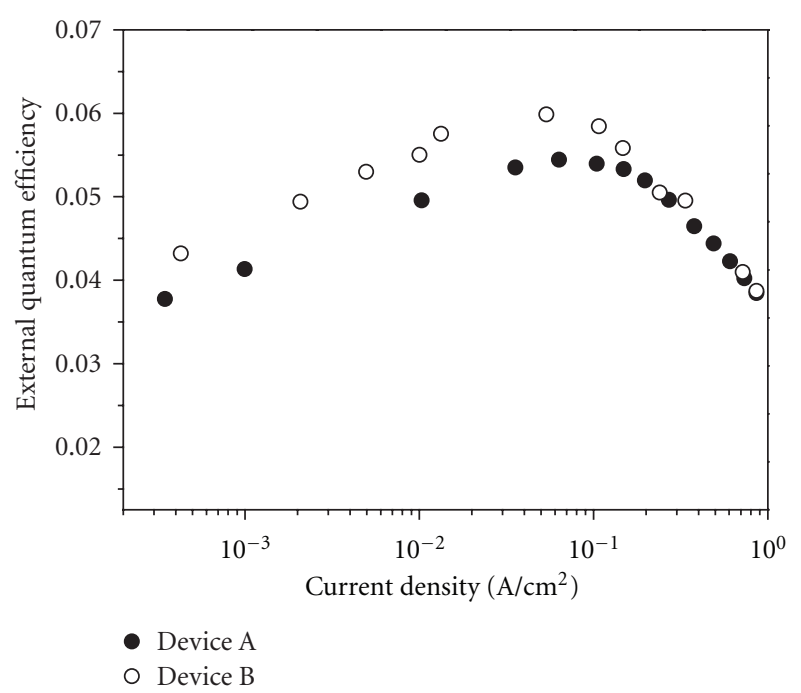

FIGURE 6: The external quantum efficiencies of Devices A and B, which are plotted against current density.

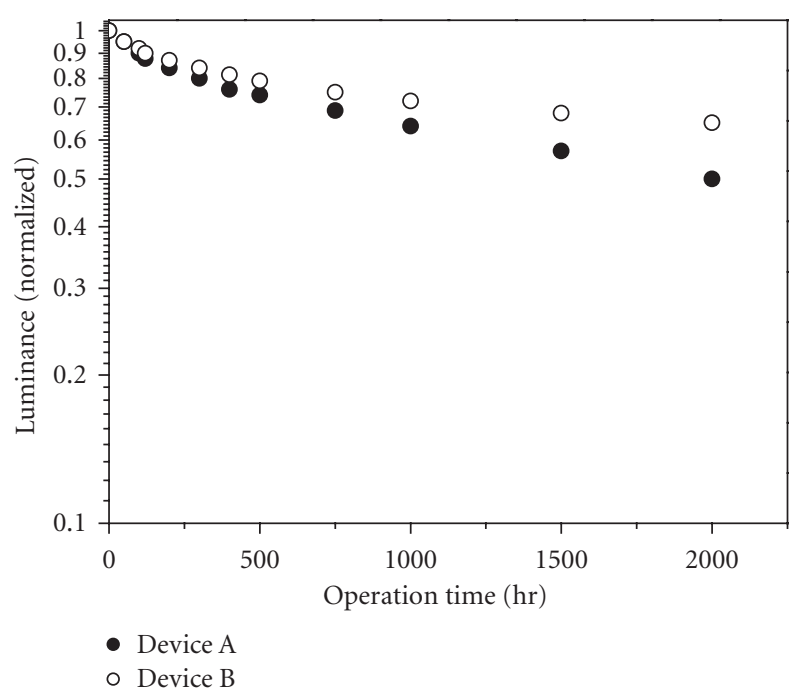

FIgURE 7: The lifetime performance of Devices A and B, which were obtained at initial luminance of $2500 \mathrm{~cd} / \mathrm{m}^{2}$ (the luminance is normalized at the initial time).

following four factors: the charge balance of holes and the electrons, the singlet exciton ratio, the photoluminescence quantum efficiency (PLQE), and the light outcoupling efficiency [11]. The charge balance and PLQE can be maximized to $100 \%$, and the singlet ratio is considered to be $25 \%$ due to the spinstatistics of the charge recombination. Thus, if the outcoupling efficiency ranges from $20 \%$ to $30 \%$, the upper limit of EQE becomes $5 \%-7.5 \%$. It is noteworthy that we obtained an almost upper EQE of 6\% (see Figure 6) in Device $\mathrm{B}$ based on the fluorescent emitter DSA-ph. The superiority of Device B to Device A is found in not only the EQE but also the device operational lifetime as shown in Figures 6 and 7, respectively. Regarding the lifetime, it is longer by about 1.3 times for Device B than for Device A. 
We consider the reason why Device B shows higher luminous, power, and external quantum efficiencies than Device A although Device B shows lower luminance at a fixed voltage than Device A. The hole and electron mobilities of ADN are in the range of $(1-5) \times 10^{-7} \mathrm{~cm}^{2} \mathrm{~V}^{-1} \mathrm{~s}^{-1}$ under external applied fields in a range of $0.5-1 \mathrm{MV} \mathrm{cm}^{-1}[12]$. The electron mobility is slightly higher than the hole mobility, for example, $4.65 \times 10^{-7} \mathrm{~cm}^{2} \mathrm{~V}^{-1} \mathrm{~s}^{-1}$ at $1 \mathrm{MV} \mathrm{cm}^{-1}$ for the former and $4.50 \times 10^{-7} \mathrm{~cm}^{2} \mathrm{~V}^{-1} \mathrm{~s}^{-1}$ for the latter. However, Ho et al. found that the electron mobility of $\mathrm{ADN}$ was one order of magnitude higher than its hole mobility [13]. Unlike Device A, BD-3 of $0.1 \mathrm{wt} \%$ concentration was codoped in EML of Device B. Such a codoping leads to decrease of electron mobility by electron trapping at BD-3 molecules and becomes comparable to the hole mobility of $\mathrm{ADN}$, resulting in a better charge balance in ADN emissive layer. In this way we understand the observed higher efficiencies of Device B than those of Device A.

The peak of the DSA-ph emission band is observed at $471.2 \mathrm{~nm}$ in Device A, while at $470.7 \mathrm{~nm}$ in Device B. Blue-shift is observed in Device B although the shift is very small. In the OLEDs with two-layer structure of ITO/ $\mathrm{NPB} / \mathrm{Alq}_{3} / \mathrm{LiF} / \mathrm{Al}$, the $\mathrm{Alq}_{3}$ emission band shows blue-shift with decreasing the $\mathrm{Alq}_{3}$ layer thickness due to optical interference effects (the recombination zone which is located at the $\mathrm{NPB} / \mathrm{Alq}_{3}$ interface tends to be closer to the metal cathode with decreasing $\mathrm{Alq}_{3}$ layer thickness) [14]. Therefore, the observed blue shift confirms the expansion of electron-hole recombination zone in Device B compared with Device A. It is also suggested that the expansion of exciton generation region contributes to lengthening the OLED operational lifetime. The reason is that, since the carriers are not confined in a limited area, Joule heating due to recombination of high density electrons and holes is avoided. Therefore we obtained the longer operational lifetime in Device B than in Device A.

\section{Conclusions}

Higher luminous and power efficiencies have been obtained at current densities below $0.1 \mathrm{~A} / \mathrm{cm}^{2}$ by codoping BD-3 into the emitting layer of ADN doped with $3 \mathrm{wt} \%$ DSA-ph when compared to the case of non-codoping. This improvement is attributed to the charge balance in emitting layer, which is obtained by reducing a higher electron mobility of ADN than the hole mobility. Additionally longer operational lifetime by 1.3 times has been achieved. This is attributed to the expansion of recombination zone, which leads to reduction of heat from recombination of high density electrons and holes in a narrow area.

\section{Acknowledgments}

This work was supported by the development fund for electronic and information industry (2010), the Key Innovation Project of Education Commission of Shanghai Municipality (12ZZ091), and the National Natural Science Foundation of China $(60906019,61136003)$.

\section{References}

[1] L. S. Hung and C. H. Chen, "Recent progress of molecular organic electroluminescent materials and devices," Materials Science and Engineering, vol. 39, no. 5-6, pp. 143-222, 2002.

[2] C. Li, M. Ichikawa, B. Wei et al., "A highly color-stability white organic light-emitting diode by color conversion within hole injection layer," Optics Express, vol. 15, no. 2, pp. 608-615, 2007.

[3] J. W. Ma, Z. Liang, C. Jin, X. Y. Jiang, and Z. L. Zhang, "Enhanced power efficiency for white OLED with $\mathrm{MoO}_{3}$ as hole injection layer and optimized charge balance," Solid State Communications, vol. 149, no. 5-6, pp. 214-217, 2009.

[4] J. M. Shi and C. W. Tang, "Anthracene derivatives for stable blue-emitting organic electroluminescence devices," Applied Physics Letters, vol. 80, no. 17, pp. 3201-3203, 2002.

[5] J. H. Lee, Y. H. Ho, T. C. Lin, and C. F. Wu, "High-efficiency fluorescent blue organic light-emitting device with balanced carrier transport," Journal of the Electrochemical Society, vol. 154, no. 7, pp. J226-J228, 2007.

[6] T. Arakane, M. Funahashi, H. Kuma et al., "Fluorescent RGB OLEDs with high performance," in Proceedings of 44th International Symposium of the Society for Information Display (SID '06), vol. 37, pp. 37-40, San Francisco, Calif, USA, June 2006.

[7] B. D. Ding, W. Q. Zhu, X. Y. Jiang, and Z. L. Zhang, "Pure blue emission from undoped organic light emitting diode based on anthracene derivative," Current Applied Physics, vol. 8, no. 5, pp. 523-526, 2008.

[8] S. Tao, Z. Peng, X. H. Zhang, and S. Wu, "Efficient and stable single-dopant white OLEDs based on 9,10-bis (2-naphthyl) anthracene," Journal of Luminescence, vol. 121, no. 2, pp. 568572, 2006.

[9] Y. Guan and L. Niu, "White organic light-emitting diodes with 9, 10-bis (2-naphthyl) anthracene," Journal of Physics: Conference Series, vol. 152, pp. 012049-012053, 2009.

[10] S. W. Wen, M. T. Lee, and C. H. Chen, "Recent development of blue fluorescent OLED materials and devices," IEEE/OSA Journal of Display Technology, vol. 1, no. 1, pp. 90-99, 2005.

[11] T. Tsutsui, "Progress in electroluminescent devices using molecular thin films," MRS Bulletin, vol. 22, no. 6, pp. 39-45, 1997.

[12] S. C. Tse, S. K. So, M. Y. Yeung, C. F. Lo, S. W. Wen, and C. H. Chen, "Experimental and theoretical demonstration on the transport properties of fused ring host materials for organic light-emitting diodes," Japanese Journal of Applied Physics, Part 1, vol. 45, no. 1, pp. 555-557, 2006.

[13] Y. H. Ho, T. C. Lin, C. F. Wu, and J. H. Lee, "High-efficiency and long-lifetime fluorescent blue organic-emitting device," in Organic Ligh Emitting Materials and Devices X, vol. 6333 of Proceedings of SPIE, p. 633303, August 2006.

[14] T. C. Lin, C. H. Hsiao, and J. H. Lee, "Study of the recombination zone of the $\mathrm{NPB} / \mathrm{Alq}_{3}$ mixed layer organic light-emitting device," in Organic Light-Emitting Materials and Devices IX, vol. 5937 of Proceedings of SPIE, pp. 59371Q-159371Q-7, August 2005. 

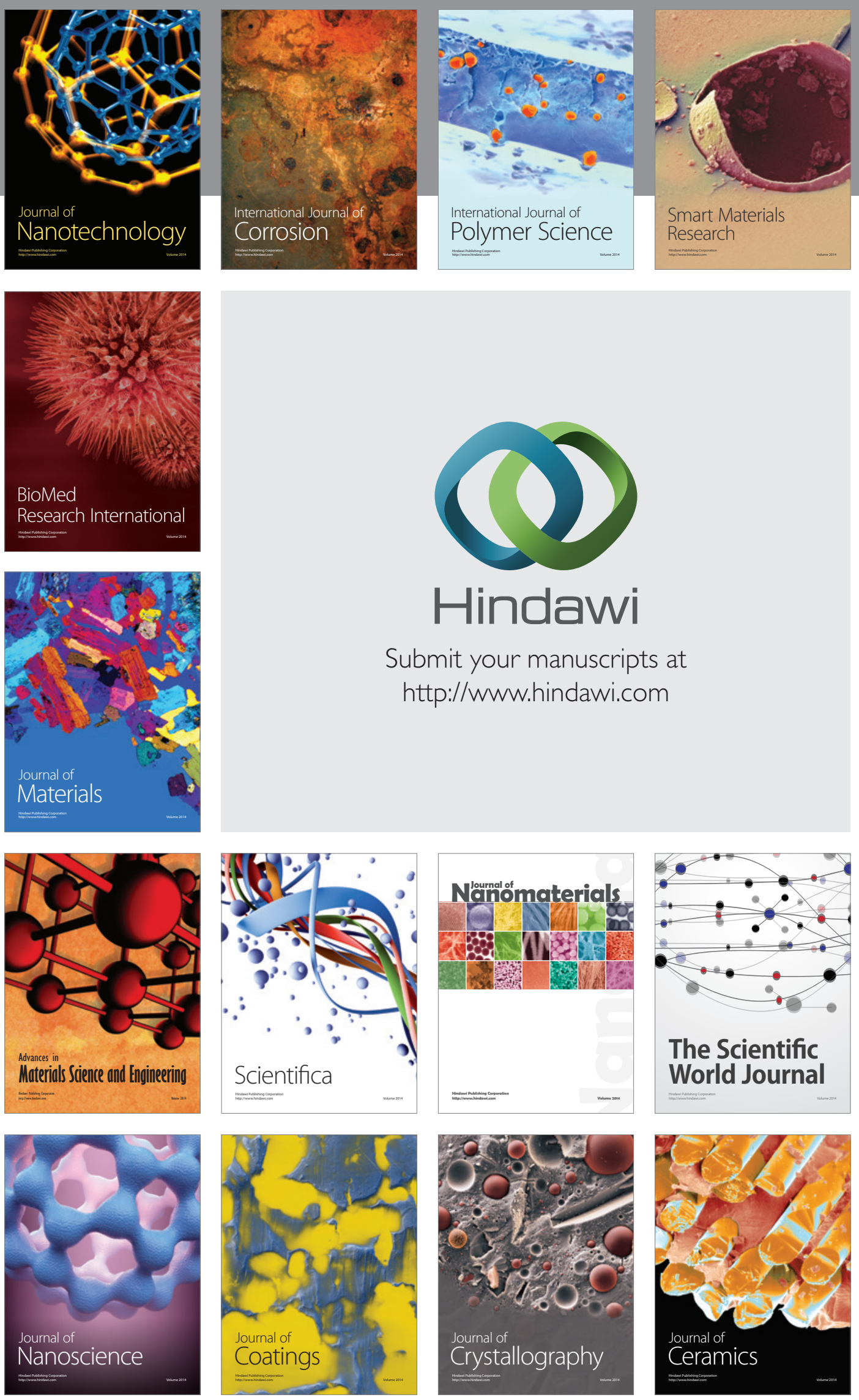

The Scientific World Journal

Submit your manuscripts at

http://www.hindawi.com

\section{World Journal}

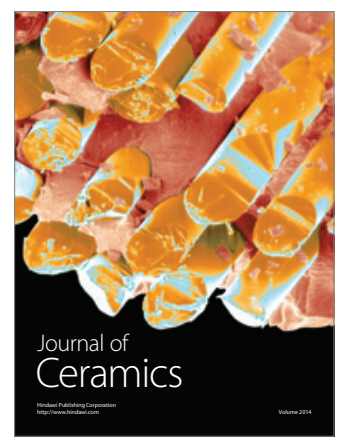

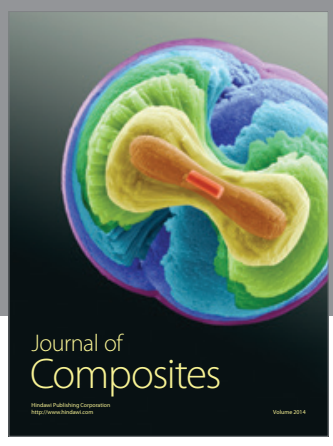
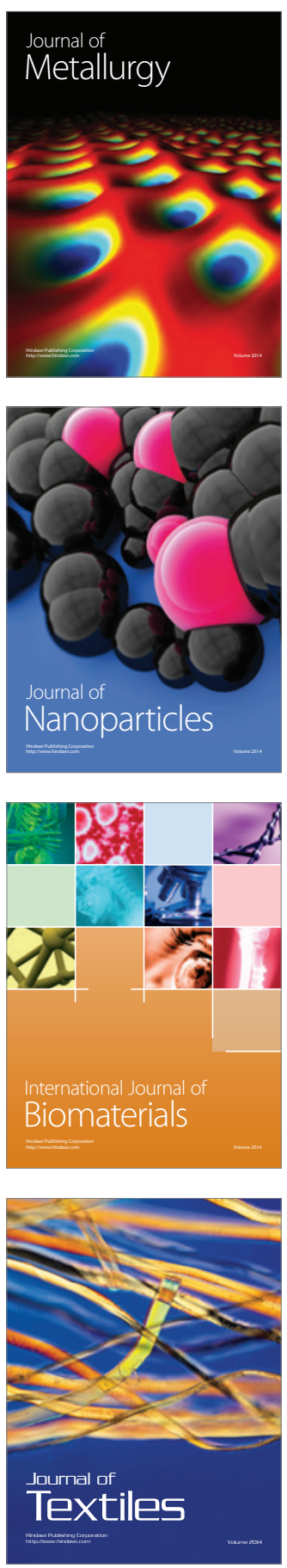